\title{
Research Paper: \\ The Relationship Between Upper Limb Function and Participation and Independence in Daily Activities of Life in People With Stroke
}

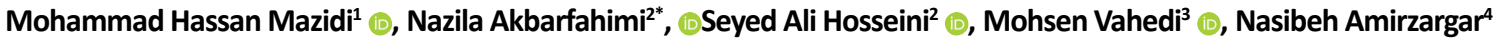

1. Department of Occupational Therapy, School of Rehabilitation Sciences, University of Social Welfare and Rehabilitation Sciences, Tehran, Iran

2. Department of Occupational Therapy, School of Rehabilitation Sciences, Rofeideh Rehabilitation Hospital, University of Social Welfare and Rehabilitation Sciences, Tehran, Iran.

3. Department of Biostatistics and Epidemiology, Iranian Research Center on Aging, Faculty of Rehabilitation Sciences, University of Social Welfare and Rehabilitation Sciences, Tehran, Iran.

4. Neurologis Rofeideh Rehabilitation Hospital, University of Social Welfare and Rehabilitation Sciences, Tehran, Iran.

\begin{tabular}{|c|c|}
\hline $\begin{array}{l}\text { Use your device to scan } \\
\text { and read the article online }\end{array}$ & \\
\hline apista & $\begin{array}{l}\text { tion and Participation and Independence in Daily Activities of Life in People With Stroke (Persian)]. Archives of Rehabilitation. } \\
\text { 2021; 22(1):86-101. https://doi.org/10.32598/RJ.22.1.3238.1 }\end{array}$ \\
\hline 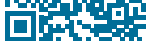 & doil'https://doi.org/10.32598/RJ.22.1.3238.1 \\
\hline
\end{tabular}

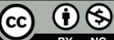

Received: 01 Aug 2020 Accepted: 12 Oct 2020 Available Online: 01 Apr 2021

Keywords:

Stroke, Participation, Upper limb function Activity of Daily Living (ADL)

\begin{abstract}
Objective Upperlimb function impairment is one of the most common consequences of stroke and a part of the motor functions, that has been considered as a necessary basis for the implementation of various of occupation and participation. The purpose of the present study is to investigate the relationship between upper extremity function and level of participation and independence in the activities of daily living in people with history of stroke in less than two years ago.

Materials \& Methods This study is non-experimental and cross-sectional. Among those referring to University of Social Welfare and Rehabilitation Sciences with a history of stroke in less than two years ago, the patients were selected by easy accessible sampling method. Upper limb performance was measured by Fogel-Meier and independency in daily living activities by the Functional Independence Scale (FIM) and participation by the Iranian Participation Questionnaire (IPQ).Data analysis was performed in SPSS V. 23 softwarebyusing Spearman correlation test.

Results The results showed that the correlation between upper limb performance and participation was a moderate direct relation $(P=0.003, r=0.315)$ and there was a strong direct $(r=0.625, P=0.00)$ correlation between upper limb performance and independence in activities of daily living.

Conclusion The relationship between upper limb function and independence in daily living activities is strong, so with further improvement and efficiency of the upper limbleadtoincreasesindependence in patients' activities of daily life. The relationship between upper limb function and participation is moderate, so to increase and improve the participation of stroke patients, in addition to upper limb mobility, other factors must be considered.
\end{abstract}

\section{Extended Abstract}

\section{Introduction}

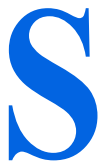

troke is one of the diseases affecting health worldwide and one of the main causes of long-term disabilities [1]. The prevalence of this disease in Iran is 372 people per 100,000 people [2].
Based on the conceptual framework of the International Classification Model of Function, Disability, and Health, health components and health-related areas are defined as body and individual social aspects along with two basic lists: 1. Functions and body structures; 2. Activities and participation [3]. According to this classification, participation is defined as dealing with different life situations, and participation limitations are problems that are experienced in dealing with different situations [4].

\section{* Corresponding Author:}

Nazila Akbarfahimi,

Address: Department of Occupational Therapy, School of Rehabilitation Sciences, Rofeideh Rehabilitation Hospital, University of Social Welfare and Rehabilitation Sciences,Tehran, Iran.

Tel: +98 (21) 22180063

E-Mail: na.akbarfahimi@uswr.ac.ir 
In addition to the simple definition of participation above, participation is a complex and broad concept at the community level that many factors, such as cultural, social, and environmental issues are considered as a deterrent or facilitator that indicate the dependence of the concept of participation on social factors and its differences in different structures and cultures [5].

About $80 \%$ of people with a history of stroke have dysfunction of the upper and lower limbs on one side of the body [6]. Restricting upper extremity movement (arm, hand, and finger) is one of the most common consequences of a stroke, which is often permanent and is a debilitating factor and affects daily life activities [7].

In occupational therapy, there is a close relationship between health and participation in acupuncture, and also the focus of occupational therapy interventions is to improve the level of individual participation in various acupunctures, such as leisure and work [8]. It is believed that maintaining a person's participation in acupuncture can help improve and maintain his health. Therefore, for occupational therapy interventions on stroke, studying participation can show the extent of the impact of stroke on a person's life and also determine the interventional method [8].

About 25 to $74 \%$ of stroke patients worldwide need some help to become independent in their daily activities or are completely dependent on caregivers to carry out their daily activities after a stroke [4]. Returning to previous activities is important for well-being and is often seen as a goal of rehabilitation; thus, it is important to examine the relationship between upper limb function and independence in daily life activities with a history of stroke $[7,9]$.

Past studies have identified the factors affecting participation and independence in daily life activities, but because of participation and its diversity in different cultures and regions, the results of studies cannot be generalized to other regions. Therefore, this study was done to investigate the relationship between the level of independence in daily life activities and participation and upper limb function in people with stroke.

\section{Materials and Methods}

This study was performed by the cross-sectional correlation method. The study sample was selected from the people referring to the treatment centers of the University of Social Welfare and Rehabilitation Sciences in 2019-20 20 , according to the research criteria, by convenience sampling method. The sample size was calculated considering a probability of the first type error of $5 \%$, a test power of
$80 \%$, using the sample volume formula for the correlation coefficient [14], concerning the correlation of 0.32 in the study of Hartman et al. [15], and 10\% loss. Inclusion criteria included the diagnosis of stroke by a specialist, the age of between 18 and 70 years, passing less than 2 years after the stroke [10], following the verbal instructions (score greater than 22 on the MMSE test) [16], no other neurological diseases, such as epilepsy and Parkinson's disease, no other orthopedic (musculoskeletal) disease, no swallowing, aphasia, and mental disorder. Exclusion criteria included no continued involvement of clients in the study and clinical instability, such as seizures and recurrent stroke. The study was approved by the Ethics Committee of Tehran University of Social Welfare and Rehabilitation Sciences (IR. USWR.REC.1398.129).

\section{Tools and Methods}

After obtaining a license from the university and occupational therapy centers and studying the medical records of stroke patients, consent was obtained from eligible individuals. Candidates were then evaluated for indicators in two sessions. In the first session, the demographic questionnaire and the functional independence measurement scale were completed, respectively, and then, in the second session, the Participation Questionnaire and Fugl-Meyer Scale were completed, respectively.

The demographic questionnaire was prepared by the researcher and included questions about the demographic characteristics of the samples and contextual variables (age, sex, marital status, type of stroke, side of the conflict, and the number of strokes).

The Iranian Participation Questionnaire was designed by Farzad et al. in 2014 to measure all aspects of participation [10]. The Fugl-Meyer scale is a tool designed by FuglMeyer based on the Branstrom and Twitchell approach to assess physical recovery after a stroke. In this study, the upper limb of this test was used [11]. The Functional Independence Scale is one of the most common tools in the rehabilitation sciences designed by Granger et al. to assess independence or dependence when performing functional daily living activities [12].

For each client, a total of 5 assessments, including demographic questionnaire, Iranian Participation Questionnaire, Fugl-Meyer Scale, Functional Independence Measurement Scale, and the Short form of Mental Status Test were completed by the researcher.

Statistical analysis of the data was done using SPSS software version 23 by Spearman test. The value of ancient D 
was used to evaluate the effect; 0.1 to 0.2 indicates a weak correlation and 0.3 to 0.4 and 0.5 to 1 indicate a moderate and strong correlation, respectively [13].

\section{Results}

The demographic characteristics of the study population are given in Table 1 . Most of the study participants were male and $29.8 \%$ of them were female. Also, $73.8 \%$ were re- tired and unemployed, most of them had secondary school education, and $83.3 \%$ were married.

The mean values of functional independence, participation, and Fugl-Meyer Scale are given in Table 2. The average scores of total participation, Fugl-Meyer Scale, and the total score of functional independence were 135.79, 26.26, and 100.17 , respectively, and their standard deviations were $40.914,20.521$, and 23.695, respectively.

Table 1. Frequency of qualitative variables $(n=84)$

\begin{tabular}{|c|c|c|}
\hline \multicolumn{2}{|c|}{ Variables } & \multirow{2}{*}{$\begin{array}{c}\text { No. (\%) } \\
59(70.2)\end{array}$} \\
\hline & Man & \\
\hline \multirow[t]{4}{*}{ Gender } & Female & $25(29.8)$ \\
\hline & Total & $84(100)$ \\
\hline & Employed & $13(15.5)$ \\
\hline & Unemployed & $33(39.3)$ \\
\hline \multirow[t]{5}{*}{ Job } & Retired & $29(34.5)$ \\
\hline & housewife & $9(10.7)$ \\
\hline & Total & $84(100)$ \\
\hline & Primary & $12(14.3)$ \\
\hline & Secondary & $47(56.0)$ \\
\hline \multirow[t]{4}{*}{ Education rate } & Masters & $12(14.3)$ \\
\hline & higher & $13(15.5)$ \\
\hline & Total & $84(100)$ \\
\hline & Ownership & $72(85.7)$ \\
\hline \multirow[t]{4}{*}{ The economic situation } & Rent & $12(14.3)$ \\
\hline & Total & $84(100)$ \\
\hline & Married & $70(83.3)$ \\
\hline & Widow & $4(4.8)$ \\
\hline \multirow[t]{3}{*}{ Marital status } & Single & $9(10.7)$ \\
\hline & divorced & $1(1.2)$ \\
\hline & Total & $84(100)$ \\
\hline
\end{tabular}


Table 2. Mean and standard deviation of quantitative variables of the studied participants $(n=84)$

\begin{tabular}{|ccccccc|}
\hline Variabels & Mean \pm SD & Min. & Max. & \multicolumn{3}{c}{ Percentiles } \\
\cline { 4 - 7 } & & & 25 & 50 & 75 \\
\hline Age & $55.8 \pm 11.99$ & 27 & 70 & 50 & 57 & 65.75 \\
\hline The motor part of functional independence & $67.54 \pm 22.104$ & 13 & 93 & 52 & 74 & 87 \\
\hline Cognitive part of functional independence & $32.83 \pm 3.53$ & 20 & 35 & 32.25 & 34 & 35 \\
\hline Total score of functional independence & $100.17 \pm 23.69$ & 47 & 126 & 85.25 & 106 & 120.75 \\
\hline Total participation score & $135.79 \pm 40.91$ & 20 & 241 & 111.50 & 137 & 156.50 \\
\hline Fugl-Meyer Scale score & $26.26 \pm 20.52$ & 2 & 64 & 9.25 & 18.50 & 45.75 \\
\hline
\end{tabular}

Rehabilitation

Table 3 indicates the correlation between the variables. According to this table, the upper extremity function variables strongly correlated with the total score of independence in daily life activities $(\mathrm{r}=0.625, \mathrm{P}<0.001)$ and the upper extremity function with moderate motor independence functional correlation $(\mathrm{r}=0.613, \mathrm{P}<0.001)$. The function of the upper limb was moderately correlated with the cognitive part of functional independence $(\mathrm{r}=0.356 \mathrm{P}=0.001)$ and participation $(\mathrm{r}=0.315, \mathrm{P}=0.003)$.

\section{Discussion and Conclusion}

The average mobility of the upper limb was 26.26 . The mobility of the upper limb was assessed based on the FuglMeyer Scale for people with a history of stroke, which was obtained from 0 to 66 . The classification of this scale is based on the study of Fugl-Meyer et al., as follows: for severe upper extremity mobility injury: $<33$, the upper extremity mobility injury: 33 to 55 , the moderate upper limb mobility injury: 56 to 62 , and mild injury group of upper limb mobility: 62 to 65 . According to this classification, the upper limb mobility injury of the participants was at a severe level.

The average degree of independence in daily life activities was 100.17. Because the items of the Functional Independence Scale are scored on a seven-point scale, the average score of the participants' independence is approximately 5.5. Accordingly, on average, participants perform various activities under supervision.

The average participation rate was 135.79 . Considering that the total score of the Iranian Participation Questionnaire was 300; thus, on average, the level of participation of

Table 3. The correlation between variables by the Spearman test

\begin{tabular}{|c|c|c|c|c|c|c|c|}
\hline Variables & 1 & 2 & 3 & 4 & 5 & 6 & 7 \\
\hline 1. Fugl-Meyer Scale score & - & & & & & & \\
\hline 2. The motor part of functional independence & $0.613^{* *}$ & _- & & & & & \\
\hline 3. Cognitive part of functional independence & $0.356^{* *}$ & $0.421^{* *}$ & _ & & & & \\
\hline 4. Total functional independence & $0.625^{* *}$ & $0.990^{* *}$ & $0.505^{* *}$ & _ & & & \\
\hline 5. Participation & $0.464 * *$ & $0.471^{* *}$ & 0.189 & $0.465^{* *}$ & - & & \\
\hline 6. Restrictions on participation & $-0.324 *$ & $-0.301 *$ & -0.147 & $-0.296^{*}$ & $-0.272 *$ & - & \\
\hline 7. Total participation & $0.315^{*}$ & $0.234^{*}$ & 0.036 & $0.226^{*}$ & $0.718^{* *}$ & $0.385^{* *}$ & _ \\
\hline
\end{tabular}

* Mean values less than 5\%; and **Mean values less than $1 \%$. 
the participants in the research was less than half of the total score of the Iranian Participation Questionnaire.

Banjai et al. showed that upper extremity sensory-motor impairment, measured by the Fugl-Meyer Scale, can determine the effect of the upper limb on self-participation in people with stroke. In contrast to the present study, they showed a strong and significant relationship between the Fugl-Meyer scale score and participation. This difference could be due to the use of the Stroke Impact Scale (SIS), hand function section, to evaluate participation. This tool is not as comprehensive as the Iranian Participation Questionnaire used in the present study [14]. Ezekel et al. showed that the relationship between hand and arm function and participation is weaker than the relationship between lower limb function or balance with participation [22]. Considering that participation is a comprehensive concept and includes the involvement of the individual in various activities and the extent of this involvement depends on various factors, motor function is only one of these factors [4]; thus, regarding the difficulty in motor function, the person can still maintain his participation with alternative solutions and the use of other factors. However, all motor functions cannot be easily replaced, including lower limb function and balance, which are necessary for movement, and the individual needs extensive changes in lifestyle and movement to compensate for the limitations in these functions. Therefore, these motor functions have a significant impact on participation [7]. However, there are other alternatives for limiting the function (mobility) of the upper limb, such as having a healthy limb on the opposite side and environmental changes and having supportive people who minimize the impact of limiting the function (mobility) of the upper limb on participation. Therefore, there is a moderate correlation between these variables. On the other hand, Andernley et al. showed that gait disorder was the most important factor in limiting participation. Participation was limited by general factors, depression, aging, dementia, which is in line with the present study [15]. Therefore, upper limb function is not the main and determining factor in the level of participation and participation of individuals in addition to motor functions (motor abilities) is limited by other factors, such as psychological and cultural factors and individual motivation.

Independence in the activities of daily life means doing them directly or indirectly without the need for the help of others [12]. Therefore, if one seeks help from others, he has reduced his independence, but participation in activities is not limited to the activities of daily living, and involvement in activities is based on the interest and desire of the individual and is not based on activities alone. Therefore, it is expected that the level of participation and independence of individuals in daily life activities is not the same. Given the non-uniformity of participation and independence in the activities of daily living and their nature, the existence of a high correlation between independence in the activities of daily living and the function of the upper limbs is justified. Akbar Fahimi et al. showed a moderate correlation between these two variables. In this study, the Bartel scale was used to evaluate the indepence of Activities Daily Living (ADL) Instrumental Activities daily Living (IADL) was also evaluated, which are more complex activities that require interaction with more factors. Thus, the effect of upper limb function on independence in these activities is reduced by other factors [7]. Jane et al. showed that the severity of incomplete upper limb paralysis is a strong component of the consequences of daily life activities, which is in line with the findings of the present study [9].

To improve future studies in this field, it is suggested to examine the effect of cultural factors on participation and independence in the daily activities of stroke clients, participation, and based on the injured side and the dominant hand.

In general, the present study showed that the average upper limb function injury of people with a history of stroke participating in the study is severe and on average, they need supervision to perform daily life activities. The relationship between upper limb function and independence in daily life activities is significantly strong; thus, as the upper limb improves and becomes more efficient, independence also increases in the patient's daily life activities. The relationship between upper limb function and significant participation is moderate; therefore, to increase and improve the involvement of stroke patients, other factors should be considered along with upper limb function.

\section{Ethical Considerations}

\section{Compliance with ethical guidelines}

The ethical principles observed in the article, such as the informed consent of the participants, the confidentiality of information, the permission of the participants to cancel their participation in the research. Ethical approval was obtained from the Research Ethics Committee of the University of Social Welfare and Rehabilitation Sciences (IR. USWR.REC.1398.129).

\section{Funding}

This study was extracted from the MSc. thesis of second author at Department Occupational Therapy of the University of Social Welfare and Rehabilitation Sciences. 


\section{Authors' contributions}

Conceptualization: Nazila Akbarfahimi, Seyed Ali Hosseini, Nasibeh Amir Zargar; Methodology: Mohammad Hassan Mazidi, Mohsen Vahedi; Investigation: Mohammad Hassan Mazidi, Nazila Akbarfahimi, Nasibeh Amir Zargar; Writing - original draft: Nazila Akbarfahimi, Mohammad Hassan Mazidi; Writing - review \& editing: All author.

\section{Conflict of interest}

The authors declared no conflict of interest. 
This Page Intentionally Left Blank 


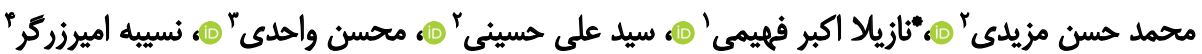

ا. كُروه كاردرمانى، دانشكده علوم توانبخشى، دانشكاه علوم توانبخشى و سلامت اجتماعى، تهراني، ايران.

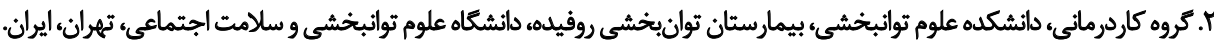

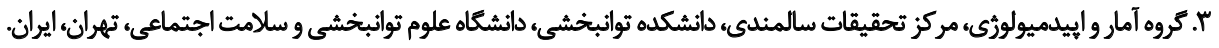

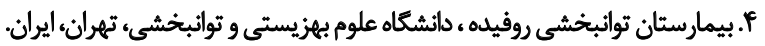

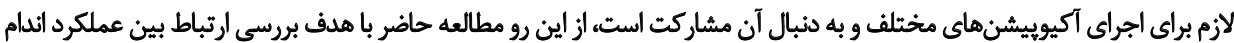

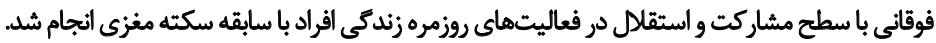

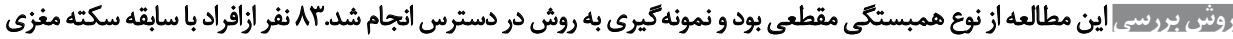

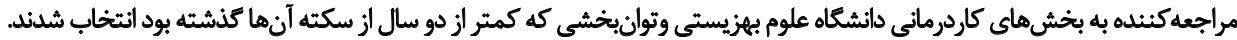

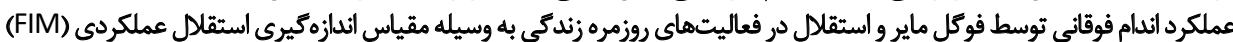

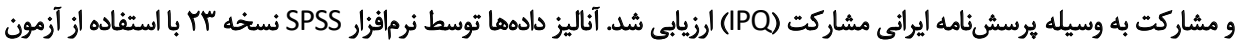

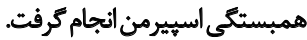

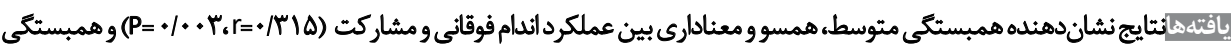

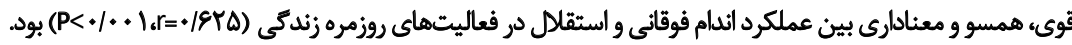

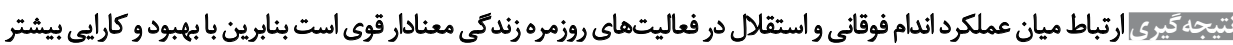

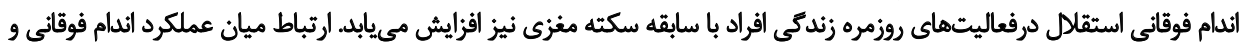

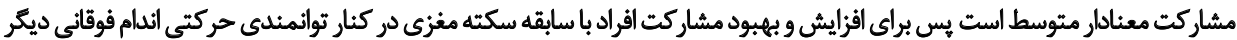

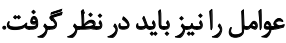

تاريخ دريافت: || تير 99" | تاريخ بذيرش: الب مهر

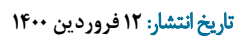

كليدوارةهها: سكته مغزئ، مشاركت،

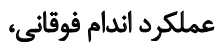

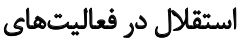

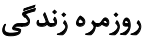

مردان، بيشتر از زنان دركير مىشوند و افراد مسن بالاى VD سال

مقدمه

كروه مستعد هستند [با].

در سكته مغزى با توجه به شريان درثير، محل و اندازه آن و و

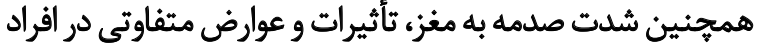

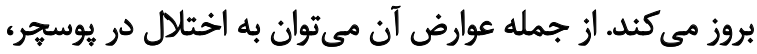

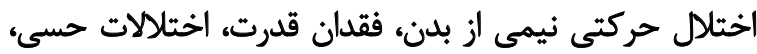

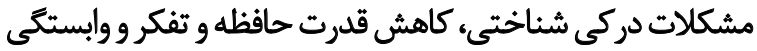

سكته مغزى يكى از بيمارىهاى تأثيركذار بر سلامتى در إنى

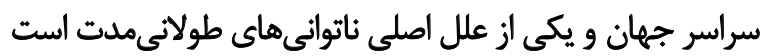

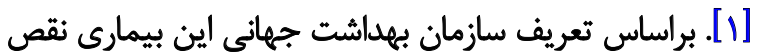

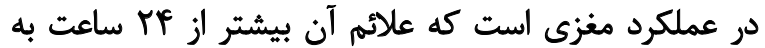

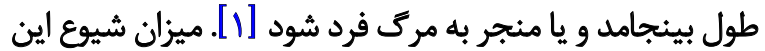

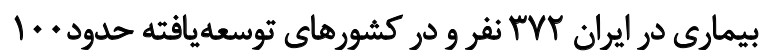

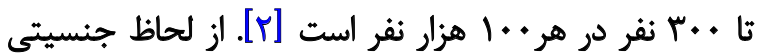


ارجاع آنان به بخشهاى كاردرمانى كزارش شده است [. 1]]. در كاردرمانى بين سلامت و مشاركت در آكويبشن ارتباط

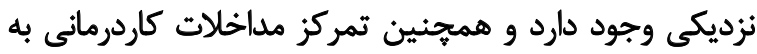

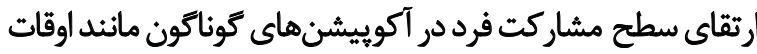

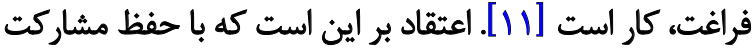

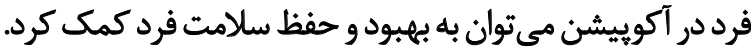

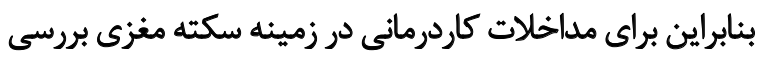

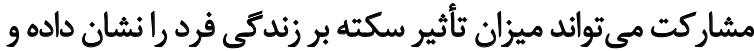

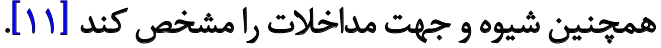

توانبخشى افراد با سابقه سكته مغزى در نتايج متغير تخمين

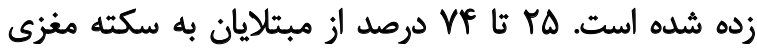

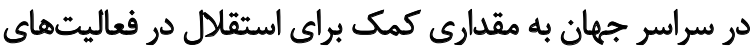

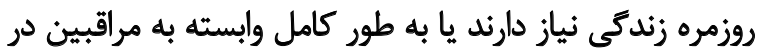

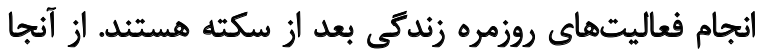

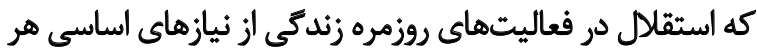

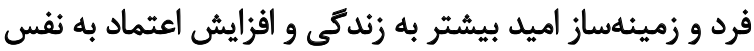

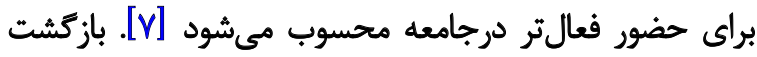

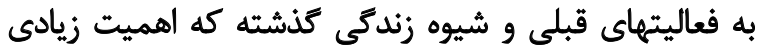

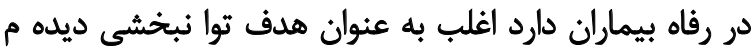

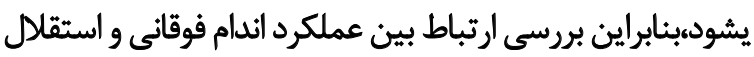
در فعالى تهاى روزمره زندئى افراد باديا سابقه سكته مغزينى حائز

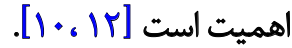

با توجه به مطالعات مرورشده از جمله اكبر فهيمى و همكاران

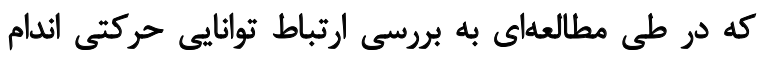

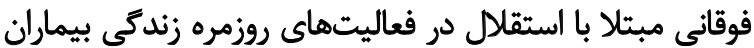

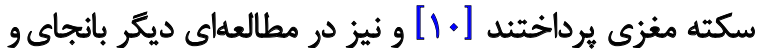

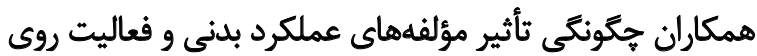

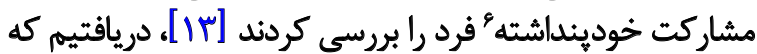

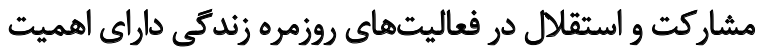

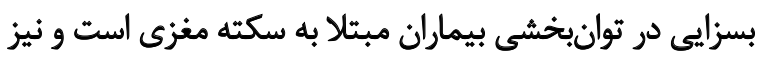

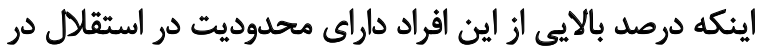

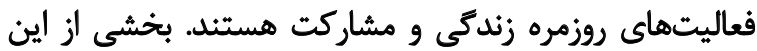

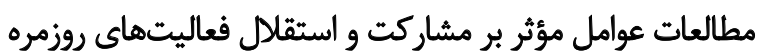

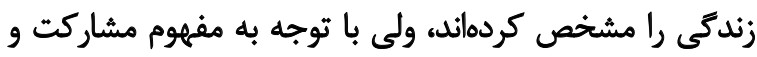

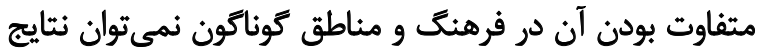

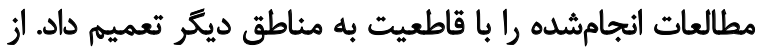

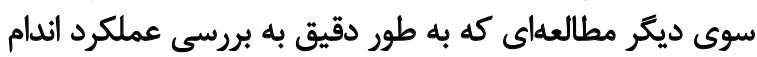

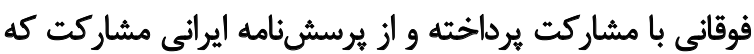

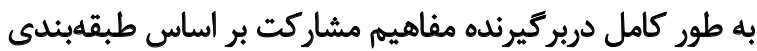

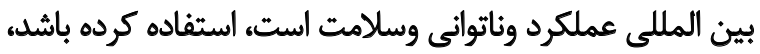

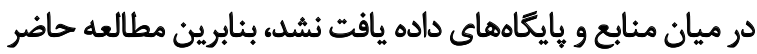

در فعاليتهاى روزمره زندكى ' اشاره كرد كه اين عوارض بر ايفاى دري

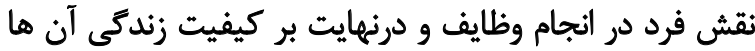

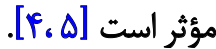

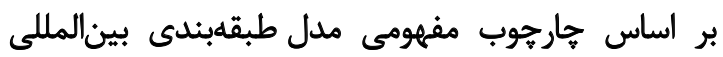

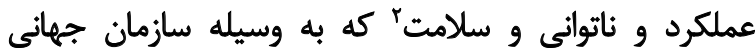

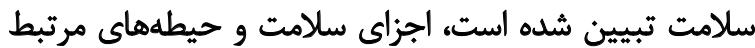

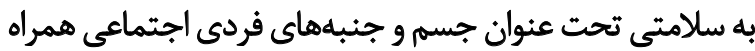

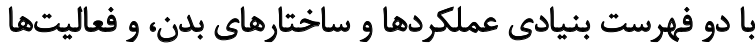

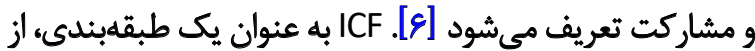

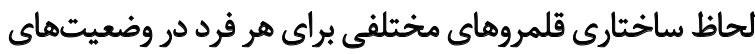

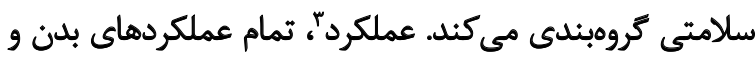

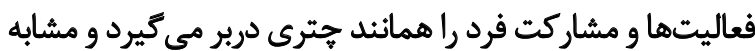

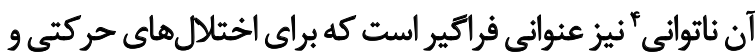

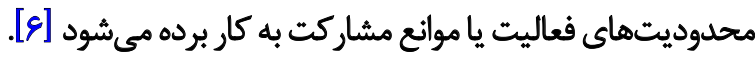

مدل زيستى روانى اجتماعى، فعاليت را به عنوان اجراى يك

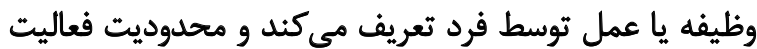

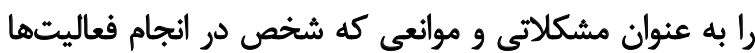

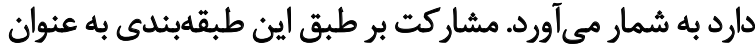

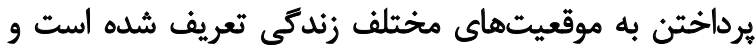

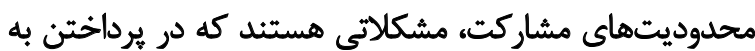
موقعيتهاي مختلف تجربه مى مشوند [Vاركيت افزون بر تعريف ساده مشاركت بيش از اين، مشاركت مفهومى

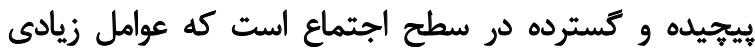

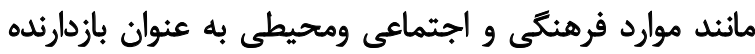

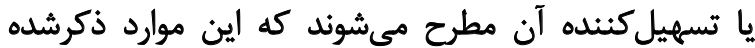

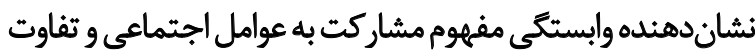

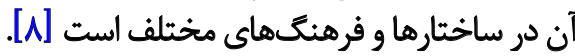

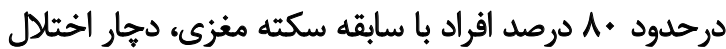

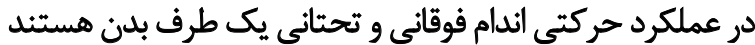

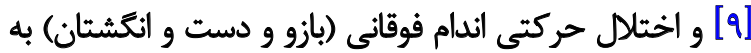

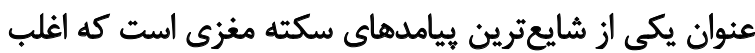

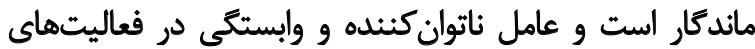

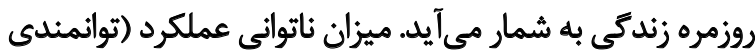

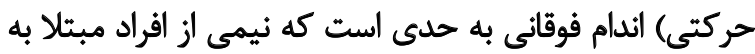

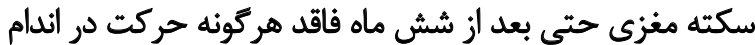

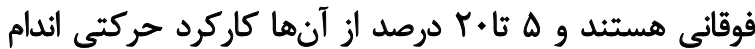

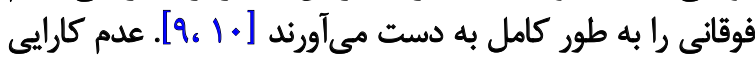
دست از شكايت اصلى بيماران سكته مغزى و از دلايل دايل عمده

1. Activity Daily Living

2. Internatinal Classification of functoining, Disability and Health (ICF)

3. Functioning

4. Disability

5. Biologicalpsycosocial model 
يرسشنامه ايرانى مشاركت توسط فرزاد و همكاران در سال

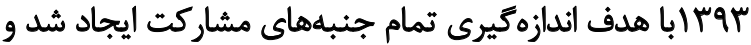

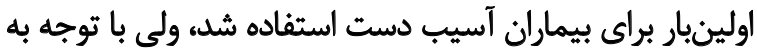

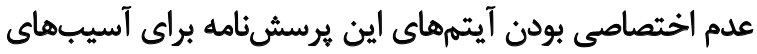

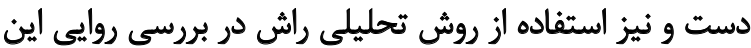

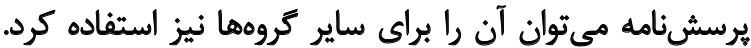

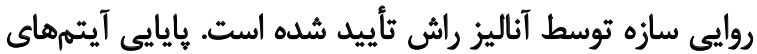

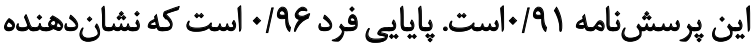

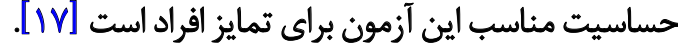

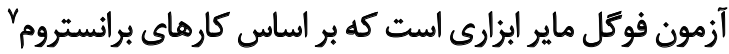

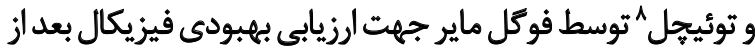

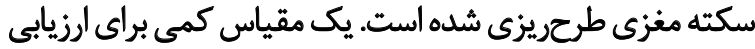

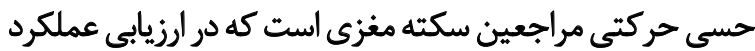

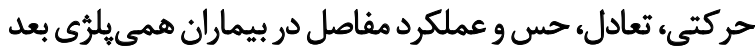

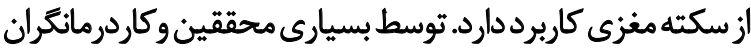

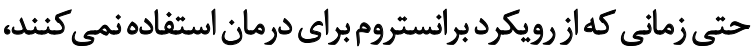

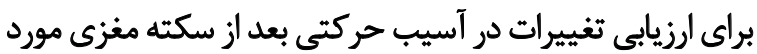

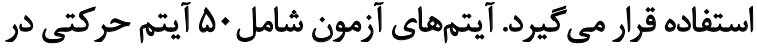

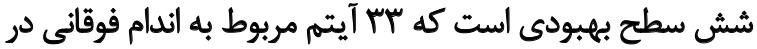

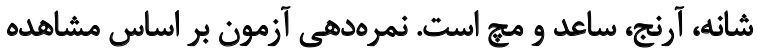

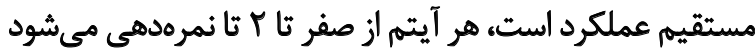

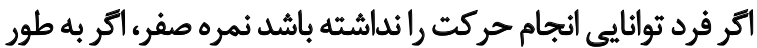

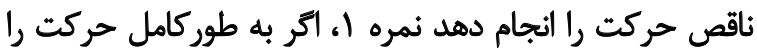

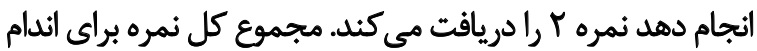

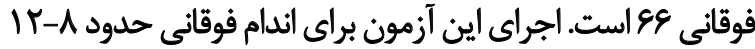

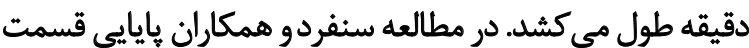

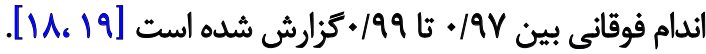

مقياس اندازميرى استقلال عملكردى يكى از ابزارهاى رايج در

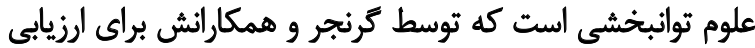

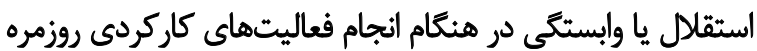

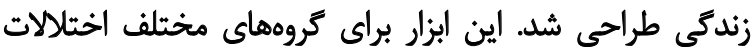

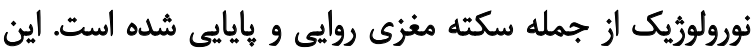

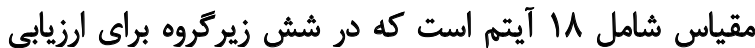

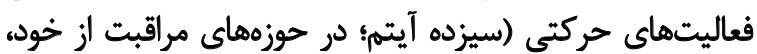

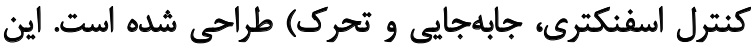

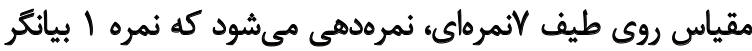

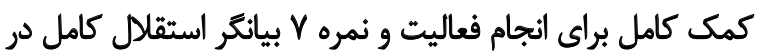

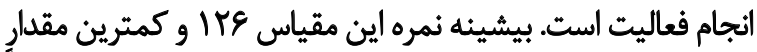

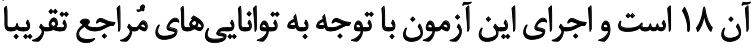

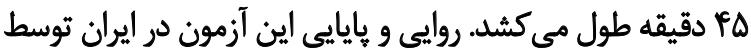

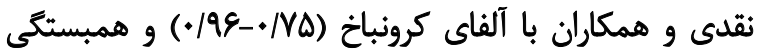

7. Brunnstrom

8. Twitchell
با هدف بررسي ارتباط بين سطح استقلال در فعاليتهائ روزمره

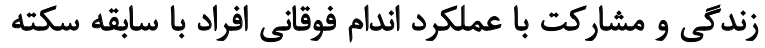
مغزى اجراشد.

يرؤوهش حاضر با توجه به مدل طبقهبندى بين المللى عملكرد

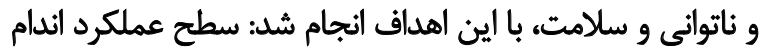

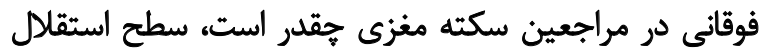

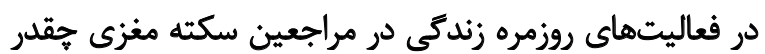

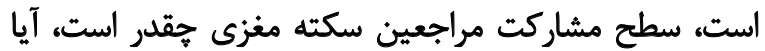

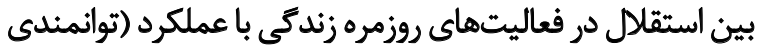

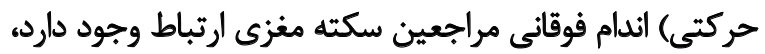

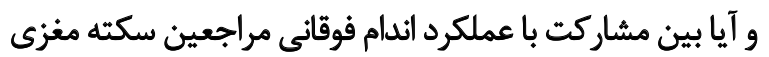

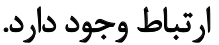

\section{ووش بروسى}

اين مطالعه به روش همبستكى مقطعى اجرا شد. نمورني

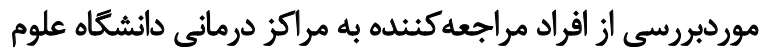

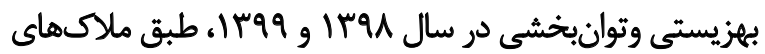

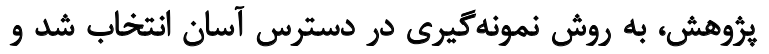

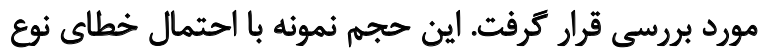

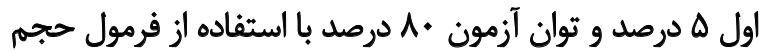

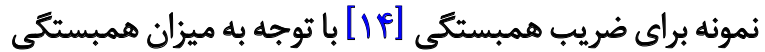

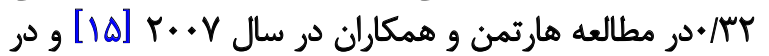

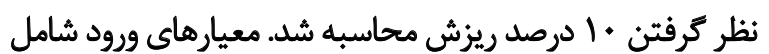

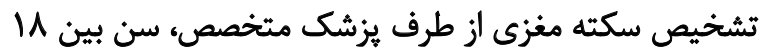

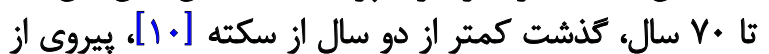

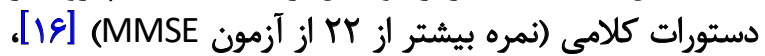

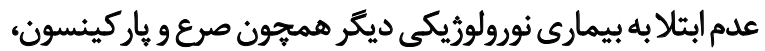

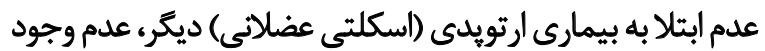

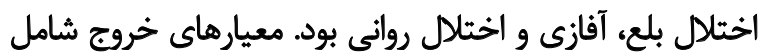

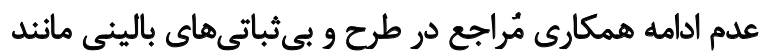

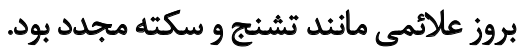

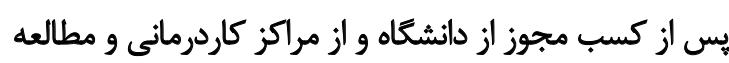

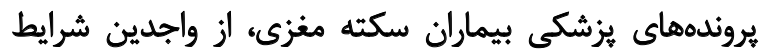

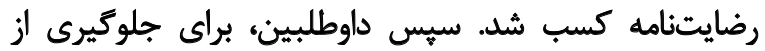

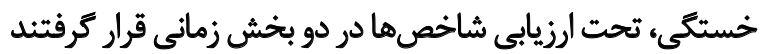

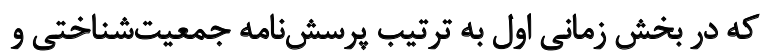

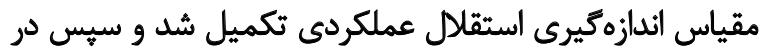

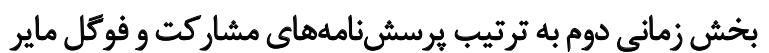

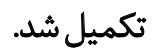

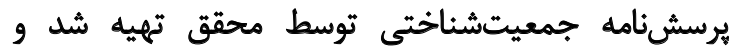

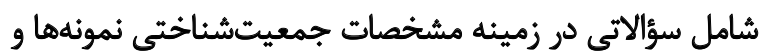

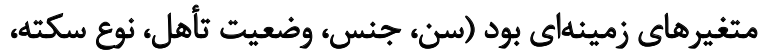
سمت دركيرى، تعداد سكته مغزى). 
جدول (. توزيع فراوانى متغيرهاي كيفى افراد مورد مطالعه (nf)

\begin{tabular}{|c|c|c|}
\hline \multirow{2}{*}{ فراواتي (مرصد) } & \multicolumn{2}{|c|}{ ويزؤىىها } \\
\hline & هرد & \\
\hline$r \Delta(Y Q / A)$ & زن & جنسيت \\
\hline$A F(1 .)$. & هجموع & \\
\hline $\mathbb{I r}(1 \Delta / \Delta)$ & شاغل & \\
\hline$\pi$ r & ييكار & \\
\hline$r q(m / \Delta)$ & بازئشسته & شغل \\
\hline $9(1 . / 7)$ & خانه دار & \\
\hline $\operatorname{Ar}(1 .)$. & مجموع & \\
\hline$\Pi(I F / M)$ & ابتدايع & \\
\hline $\operatorname{Pv}(\Delta \% / \%)$ & متوسطه & \\
\hline$M(I F / Y)$ & كارششاسى & ميزان تحصيلات \\
\hline$\Psi(1 \Delta / \Delta)$ & بالاتر & \\
\hline Af $(1 \cdot)$. & مجموع & \\
\hline$V(A \Delta M)$ & تملكى & \\
\hline$I r(I F / M)$ & استيجارى & وضعيت اقتصادى \\
\hline$A f(1 .)$. & مجموع & \\
\hline$V \cdot(A r / N)$ & متأهل & \\
\hline$\varphi(f / A)$ & بيوه & \\
\hline $9(1 . M)$ & مجرد & وضعيث تاهل \\
\hline$I(N / T)$ & مطلقه & \\
\hline $\operatorname{Ar}(1.0)$ & هجموع & \\
\hline
\end{tabular}

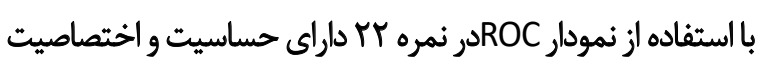

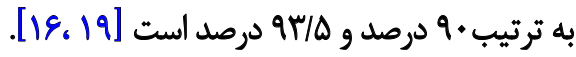

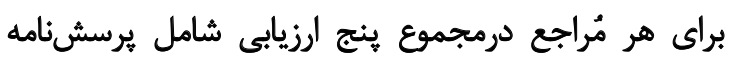

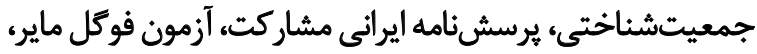

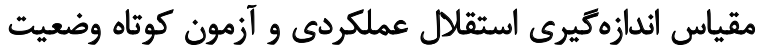

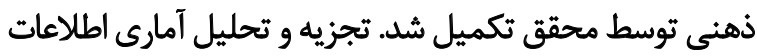

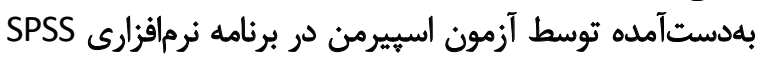

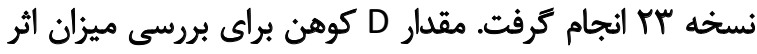

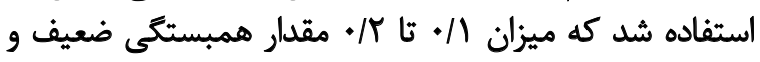

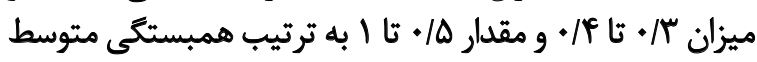

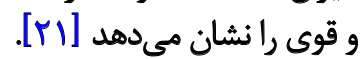

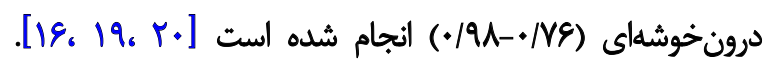

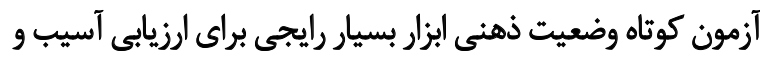

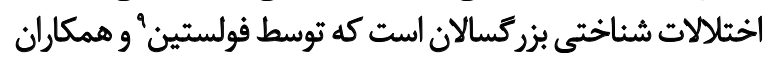

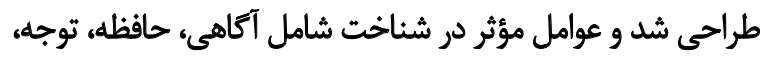

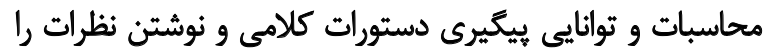

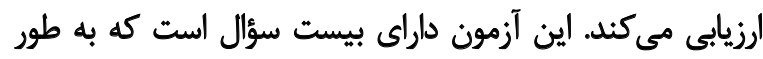

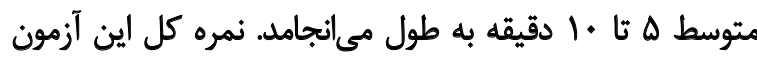

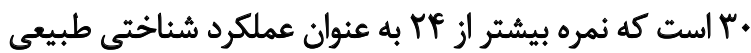

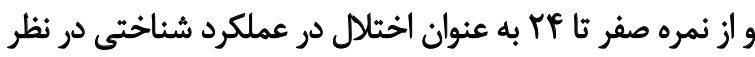

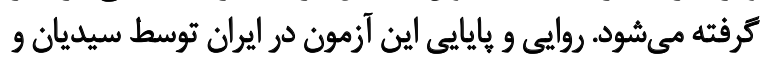

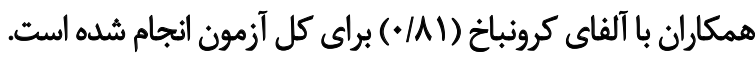


جدول r. ميانكين و انحراف معيار متغيرهاى كمى شركت كنيدكان موردمطالعه (n=AF)

\begin{tabular}{|c|c|c|c|c|c|c|}
\hline \multicolumn{3}{|c|}{ صدكها } & \multirow{2}{*}{ حداكثر } & \multirow{2}{*}{ حداقل } & \multirow{2}{*}{ ميانكين دانحراف معيار } & \multirow{2}{*}{ متغيرها } \\
\hline yo & $\Delta \cdot$ & ro & & & & \\
\hline$\varepsilon \Delta / V \Delta$ & $\Delta V$ & ه. & r. & m & $\Delta \Delta / \lambda \pm 11 / 99$ & 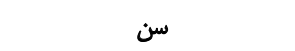 \\
\hline AV & $n$ & $\Delta r$ & צ" & ir & $g V / \Delta F \pm T r / 1 \cdot F$ & بخش حركتى استقلال عملكردى \\
\hline ro & $\pi$ & $r T / r \Delta$ & ro & r. & $\Pi r / \Lambda r \pm r / \Delta T q$ & بخش شئاختى استقلال عملكردى \\
\hline IT.Mo & 1.8 & $A \Delta / T \Delta$ & ire & pr & $1 . . / 1 \gamma \pm T r / e q \Delta$ & نمره كل استقالل عملكردى \\
\hline $1 \Delta \& / \Delta$. & irv & $111 / \Delta$. & mel & r. & $1 r \Delta / M \pm \pm r$ - $q 1 \varphi$ & نمره كل مشاركت \\
\hline$r \Delta / v \Delta$ & WA. & q/TO & af & r & TE/TEET/ATI & فوكل ماير \\
\hline
\end{tabular}

ميزان توانمندى حركتى اندام فوقانى به طور متوسط برابر با

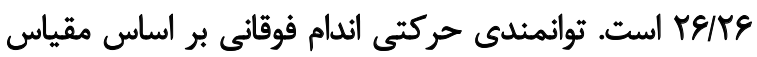

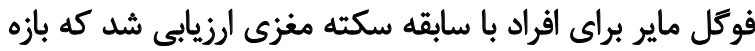

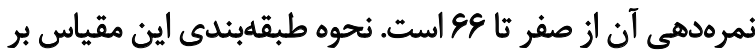

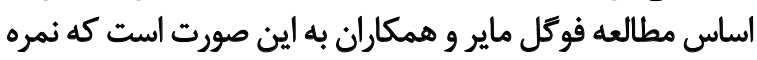

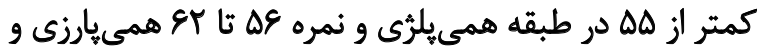

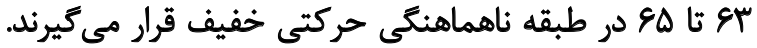

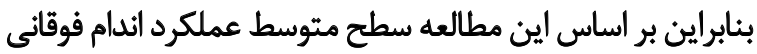

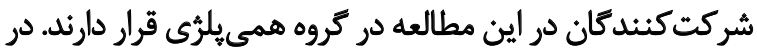

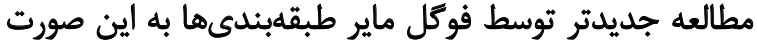

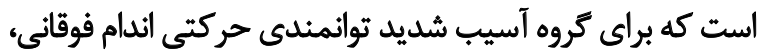

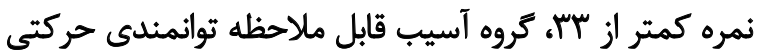

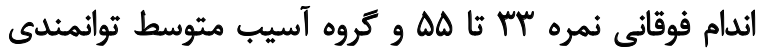

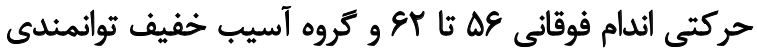

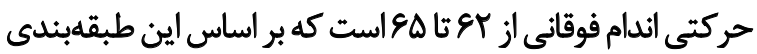

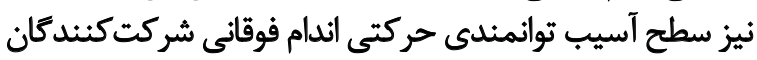

مشخصات جمعيتى جامعه مورد مطالعه در جدول شماره

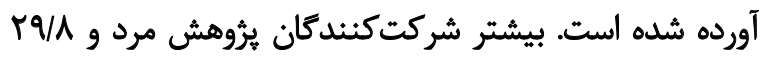

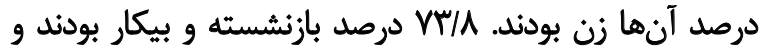

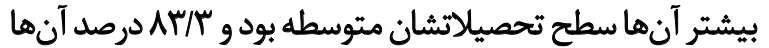

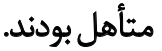

شاخصهاى آمارى استقلال عملكردى و مشاركت و فوركل

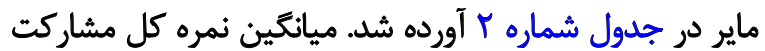

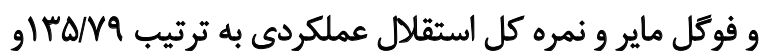

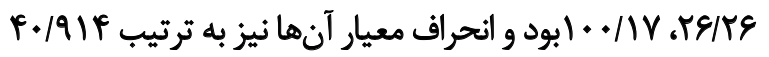
و

در جدول شماره ب بررسى همبستكى بين متغيرها آمده است.

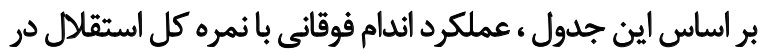

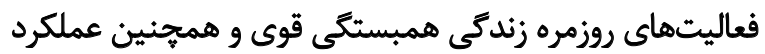

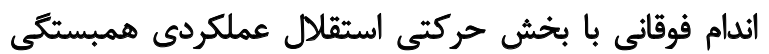

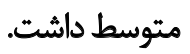

جدول با. بررسى همبستكى بين متغيرها به وسيله آزمون اسيّيرمن

\begin{tabular}{|c|c|c|c|c|c|c|c|}
\hline$\checkmark$ & 7 & $\bullet$ & $\varepsilon$ & 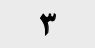 & r & 1 & مثغير ها \\
\hline & & & & & & - & فوكل ماير \\
\hline & & & & & - &.$/ 81$ (re* & بخش حركتى استقلال عملكردى \\
\hline & & & & - &.$/ M T I^{*}$ &.$/ T \Delta \mathscr{V}^{* *}$ & بخش شئختى استقلال عملكردى \\
\hline & & & - & $\cdot / \Delta \cdot \theta^{* *}$ & $. / 99 . *$ & . $/ 8 T Q^{* *}$ & السيقالال عملكردى كل \\
\hline & & - &.$/ 48 \theta^{* *}$ &.$/ 149$ & $. / p v=$ & - pegen & هشاركت \\
\hline & - & $-* / r r^{*}$ & $-+/$ ren & $-o / 1 P r$ & $-* \pi+1^{*}$ &.$- / M T p e$ & هحدوديتهاي مشاركت \\
\hline - & $+/ \% \Delta \Delta^{* *}$ & $\cdot M \mathrm{~N}^{* * *}$ & •/MYE* & (1. ( &.$/ \pi \pi^{c}$ &.$/ 10^{*}$ & مشاركت كل \\
\hline
\end{tabular}


افراد حمايت كننده وجود دارد كه ميزان تأثير محدوديت عملكرد

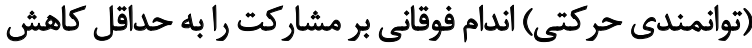

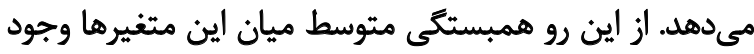

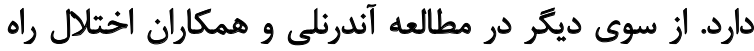

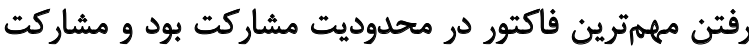

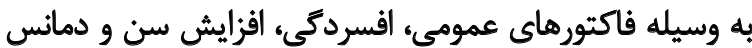

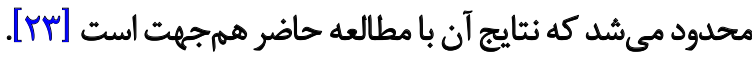

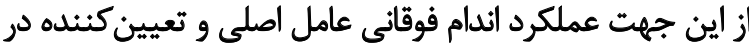

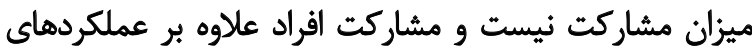

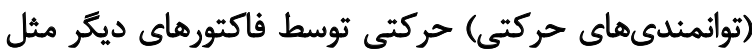

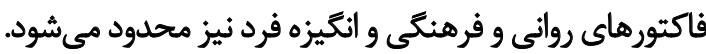

نتايج همبستخى بين متغيرهاى عملكرد اندام فوقانى و استقلال

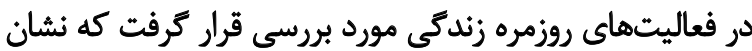

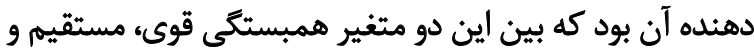

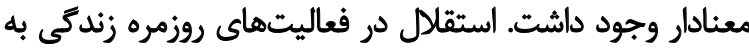

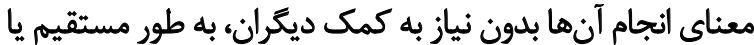

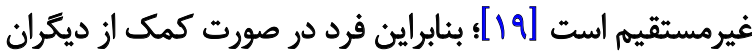

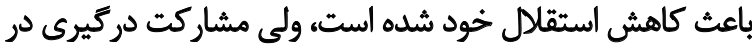

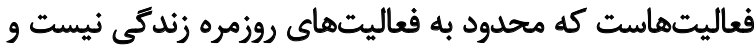

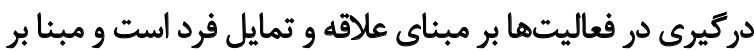

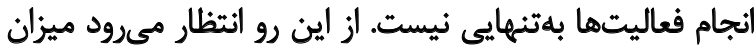

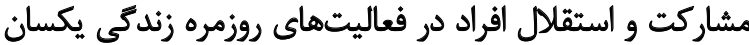

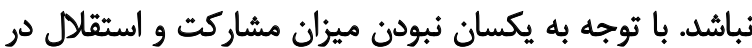

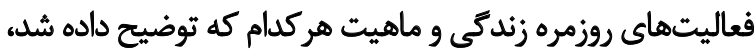

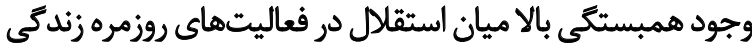

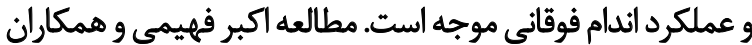

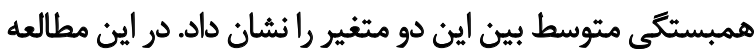

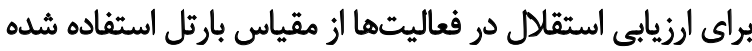

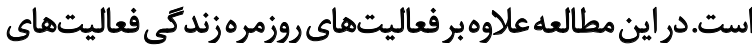

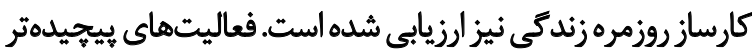

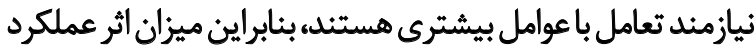

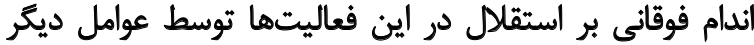

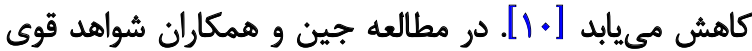

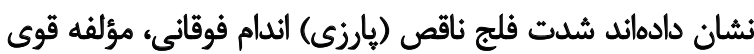

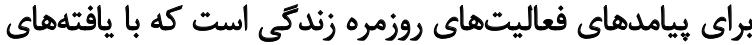

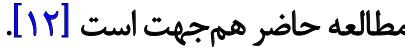

\section{نتيجلهيرى}

به طور كلى مطالعه حاضر نشان داد متوسط آسيب عملكرد

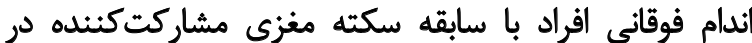

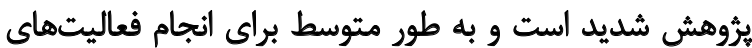

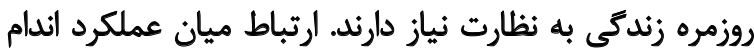

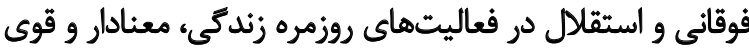

درمجموع در كروه شديد قرار مي كيرند.

ميزان استقلال در فعاليتها روزمره زندكى به طور متوسط إندائ

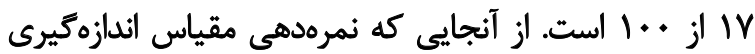

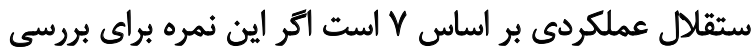

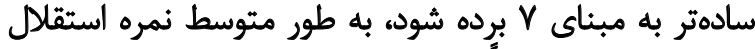

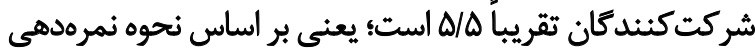

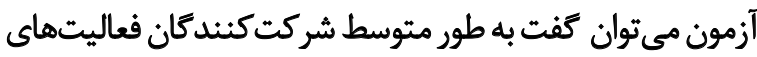
مختلف را با نظارت انجام مى مهند.

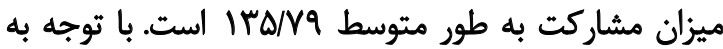

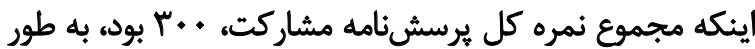

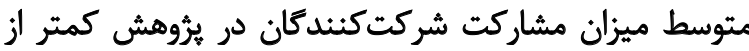
نصف نمره كل يرسشنان مشاركه مشاركث است.

نتايج همبستكى بين متغيرهاى عملكرد اندام فوقانى و و مثني

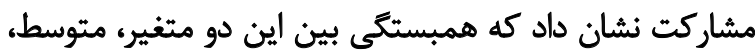

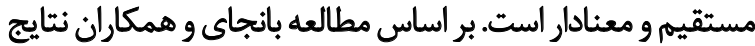

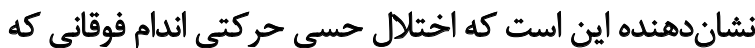

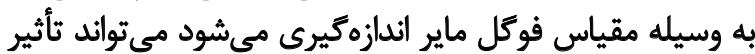

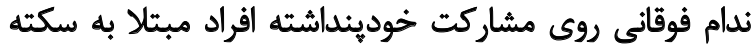

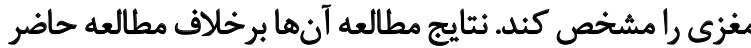

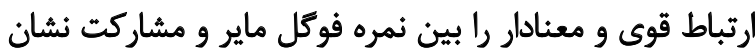

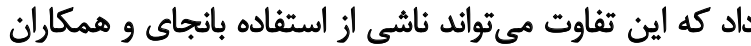

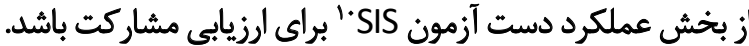

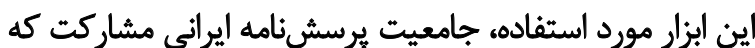

در مطالعه حاضر استفاده شده است راندارد [باد إن]

در مطالعه ازيكل و همكاران ارتباط ميان عملكرد دست و بازو

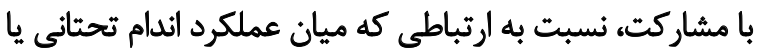

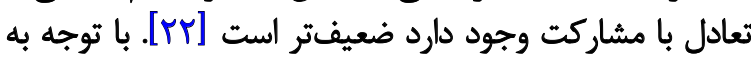

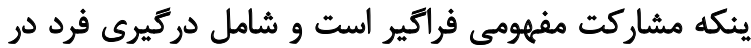

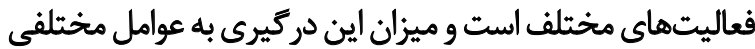

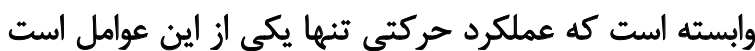

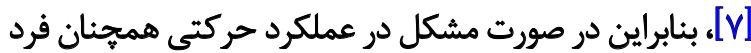

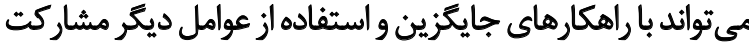

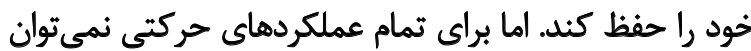

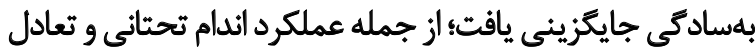

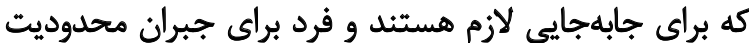

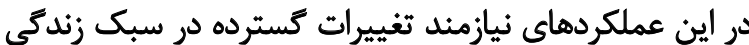

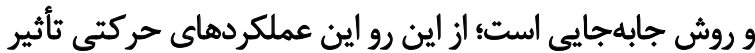

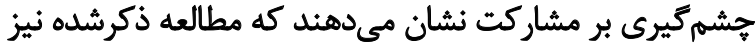

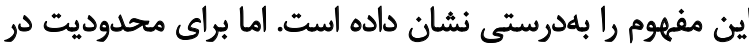

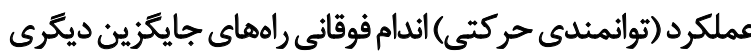

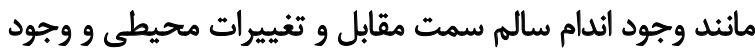

10. Stroke Specifc Scale 
است، بنابراين با بهبود و كارايى بيشتر اندام فوقانى استقلال در

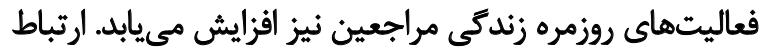

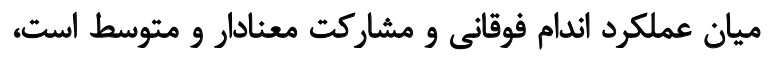

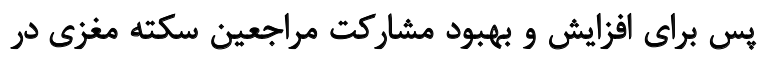

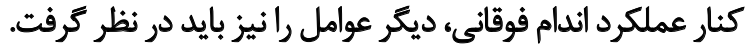

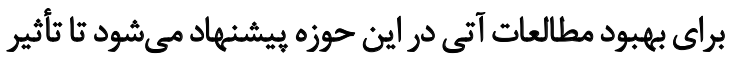

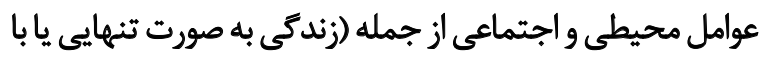

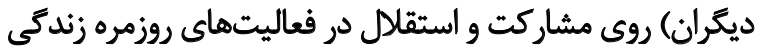

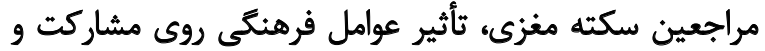

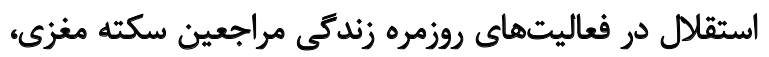

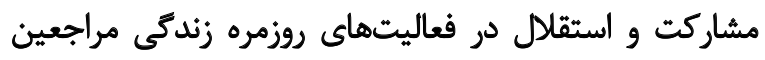

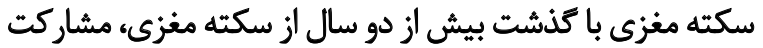

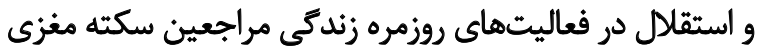

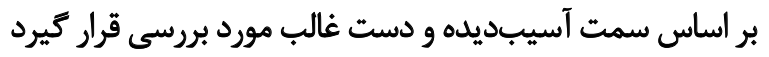

مالاحظات اخلاقى

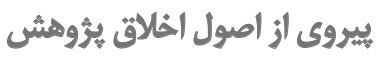

در اجراى برؤهش ملاحظات اخلاقي مطابق بادستور العمل كميته

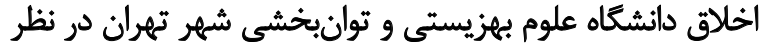

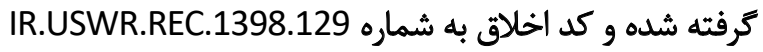

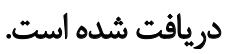

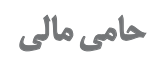

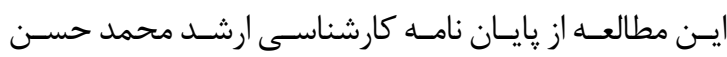

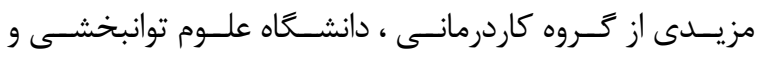

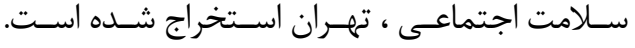

مشاركت نويسند مَان مفهوم سازى: نازيلا اكبرفهيمى، سيدعلى حسينى، نسيبه

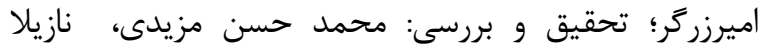

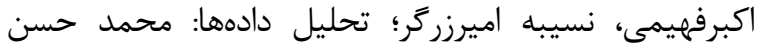

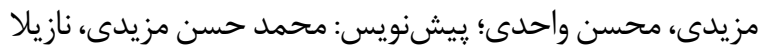
اكبرفهيمى؛ ويراستارى و نهايى سازى: تمامى نويسند محكان. تعارض منافع بنابر اظهار نويسندكان اين مقاله تعارض منافع ندارد 


\section{References}

[1] Hofheinz M, Mibs M, Elsner B. Dual task training for improving balance and gait in people with stroke. The Cochrane Database of Systematic Reviews. 2016; 2016(10):CD012403. [DOI:10.1002/14651858.CD012403] [PMCID]

[2] Saberi A, Ghayeghran AR, Janeshin S, Biazar G, Kazemnezhad E. Opium consumption prevalence among patients with ischemic stroke compared with healthy individuals in Iran. International Journal of High Risk Behaviors and Addiction. 2017; 6(1):45-65. [DOI:10.5812/ijhrba.27264]

[3] Long BC, Phipps WJ, Cassmeyer VL. Adult nursing: A nursing process approach. Amsterdam: Elsevier Health Sciences; 1995. https://books.google.com/books?id=

[4] Pedretti LW, Early MB, editors. Occupational therapy: Practice skills for physical dysfunction. St. Louis, MO: Mosby; 2001.

[5] Radomski MV, Latham CA, editors. Occupational therapy for physical dysfunction. Philadelphia: Lippincott Williams \& Wilkins; 2008. https://books.google.com/books?h

[6] International classification of functioning, disability and health: ICF, (2001). https://www.worldcat.org/title/designing-clinicalresearch/oclc/868930845? referer=di\&ht=edition

[7] Singam A, Ytterberg C, Tham K, von Koch L. Participation in complex and social everyday activities six years after stroke: predictors for return to pre-stroke level. PloS One. 2015; 10(12):e0144344. [DOI:10.1371/journal.pone.0144344] [PMID] [PMCID]

[8] World Health Organization 2001. The International Classification of Functioning, Disability and Health (ICF). Geneva: WHO. http://www.who.int/classifications/icf/en/

[9] Pollock A, Farmer SE, Brady MC, Langhorne P, Mead GE, Mehrholz J, et al. Interventions for improving upper limb function after stroke. Cochrane Database of Systematic Reviews. 2014; 11(11):43-50. [DOI:10.1002/14651858.CD010820.pub2] [PMID] [PMCID]

[10] Akbarfahimi M, Karimi H, Rahimzadeh Rahbar S, Ashaeri H, Faghehzadeh S. [The relationship between motor function of hemiplegic upper limb and independency in activities of daily of living in stroke patients in Tehran (Persian)]. Koomesh. 2011; 12(3):236-43. http://koomeshjournal.semums.ac.ir/article1-1225-en.html

[11] Fallahpour M, Jonsson H, Joghataei MT, Kottorp A. Impact on Participation and Autonomy (IPA): Psychometric evaluation of the Persian version to use for persons with stroke. Scandinavian Journal of Occupational Therapy. 2011; 18(1):59-71. [DOI:10.3109/11038121003628353] [PMID]

[12] Veerbeek JM, Kwakkel G, van Wegen EE, Ket JC, Heymans MW. Early prediction of outcome of activities of daily living after stroke: A systematic review. Stroke. 2011; 42(5):1482-8 [DOI:10.1161/STROKEAHA.110.604090] [PMID]

[13] Banjai RM, Freitas SM, Silva FP, Alouche SR. Individuals' perception about upper limb influence on participation after stroke: An observational study. Topics in Stroke Rehabilitationn. 2018; 25(3):174-9. [DOI:10.1080/10749357.2017.1406177] [PMID]
[14] Hulley SB CS, Browner WS, Grady D, Newman TB. Designing clinical research: an epidemiologic approach. 4 ed. Lippincott Williams \& Wilkins; 2013. https://www.worldcat.org/title/ designing-clinical-research/oclc/868930845? referer=di\&ht=edi tion

[15] Hartman-Maeir A, Soroker N, Ring H, Avni N, Katz N. Activities, participation and satisfaction one-year poststroke. Disability and Rehabilitation. 2007; 29(7):559-66. [DOI:10.1080/09638280600924996] [PMID]

[16] Seyedian M, Fallah M, Nourouzian M, Nejat S, Delavar A, Ghasemzadeh H. Validity of the Farsi version of mini-mental state examination. Journal of Medical Council of Iran. 2008; 25(4):408-14. aspx?id $=133666$ https://www.sid.ir/en/journal/ViewPaper

[17] Farzad M, Asgari A, Dashab F, Layeghi F, Karimlou M, Hosseini SA, et al. Does disability correlate with impairment after hand injury? Clinical Orthopaedics and Related Research $® . ~ 2015$; 473(11):3470-6. [DOI:10.1007/s11999-015-4228-7] [PMID] [PMCID]

[18] de Bode S, Fritz SL, Weir-Haynes K, Mathern GW. Constraintinduced movement therapy for individualsafter cerebral hemispherectomy: A case series. Physical Therapy. 2009; 89(4):361-9. [DOI:10.2522/pti.20070240] [PMID] [PMCID]

[19] Radomski MV, Latham CA, editors. Occupational therapy for physical dysfunction. Philadelphia: Lippincott Williams \& Wilkins; 2008. https://books.google.com/books?hl

[20] Naghdi S, Ansari NN, Raji P, Shamili A, Amini M, Hasson S Cross-cultural validation of the Persian version of the Functional Independence Measure for patients with stroke. Disability and Rehabilitation. 2016; 38(3):289-98. [DOI:10.3109/09638288.201 5.1036173] [PMID]

[21] Cohen J. Statistical power analysis for the behavioral sciences (Revised ed.). Hillsdale, NJ: Lawrence Earlbaum Associates. https://lab.rockefeller.edu/cohenje/PDFs/159CohenBigFishLittle FishTheSciences1989.pdf

[22] Ezekiel L, Collett J, Mayo NE, Pang L, Field L, Dawes H. Factors associated with participation in life situations for adults with stroke: A systematic review. Archives of Physical Medicine and Rehabilitation. 2019; 100(5):945-55. [DOI:10.1016/j. apmr.2018.06.017] [PMID]

[23] Andrenelli E, Ippoliti E, Coccia M, Millevolte M, Cicconi B, Latini L, et al. Features and predictors of activity limitations and participation restriction 2 years after intensive rehabilitation following first-ever stroke. European Journal of Physical and Rehabilitation Medicine. 2015; 51(5):575-85. https://www.researchgate. net/profile/Marianna-Capecci/publication/271537284_Features_and_predictors_of_activity_limitations_and_participation_restriction_2 years_after_intensive rehabilitation following_first-ever_stroke/links/552e65e10cf2d4950717ec09/ Features-and-predictors-of-activity-limitations-and-participationrestriction-2-years-after-intensive-rehabilitation-following-firstever-stroke.pdf 
This Page Intentionally Left Blank 